\title{
Uma taxonomia de Visual Analytics em Sistemas de Apoio à Decisão
}

\author{
Gustavo Romão Gonzales ${ }^{1}$, Lívia Castro Degrossi ${ }^{2}$, Flávio Horita ${ }^{1}$ \\ ${ }^{1}$ Centro de Matemática, Computação e Cognição - Universidade Federal do ABC (UFABC) \\ Santo André - SP - Brazil \\ ${ }^{2}$ Fundação Getulio Vargas \\ São Paulo - SP - Brazil \\ \{gustavo.romao, flavio.horita\}@ufabc.edu.br \\ livia.degrossiefgv.br
}

\begin{abstract}
Decision Support Systems (DSS) are part of the daily life of all environments, acting in support of the decision-making process, assisting the data usage as a basis, enabling more efficient and advantageous decisions. The increase in the volume of digitally produced data brings countless opportunities and organizations have improved their DSS as a means of discovering new information hidden in this data. As a means of solving the problem of data overload with one of the difficulties, Visual Analytics (VA) is applied supporting use and exploration of this data. However, the implementation of VA in a DSS is not a trivial task, currently requiring development and evaluation techniques. The present work aims to present a VA taxonomy in SAD. To this end, the project adopted a systematic methodology for the establishment of taxonomy. The main contribution of this project is to present a taxonomy that can classify and describe the DSS architectures that implements VA.
\end{abstract}

Resumo. Sistemas de Apoio à Decisão fazem parte do dia a dia de todos os ambientes, atuando no suporte ao processo de tomada de decisão, auxilia na utilização de dados como embasamento, possibilitando decisões mais eficientes e vantajosas. $O$ aumento do volume de dados produzidos digitalmente traz inúmeras oportunidades e as organizações tem aperfeiçoado seus SAD como meio de descobrir novas informações ocultas nestes dados. Como meio de resolver o problema de sobrecarga de dados com uma das dificuldades, o Visual Analytics (VA) é aplicado no auxílio do uso e exploração desses dados. No entanto, a implementação de VA em um SAD não é uma tarefa trivial, exigindo atualmente técnicas de desenvolvimento e avaliação. O presente trabalho tem como objetivo apresentar uma taxonomia de VA em SAD. Para tanto, o projeto adotou uma metodologia sistemática para o estabelecimento da taxonomia. A principal contribuição deste projeto é apresentar uma taxonomia que possa classificar e descrever as arquiteturas dos SAD que implementam VA.

\section{Introdução}

O gerenciamento e tomada de decisão evoluiu com a utilização dos Sistemas de Apoio à Decisão (SAD). Desde meados da década de 70, as organizações governamentais ou não 
utilizam o conhecimento empírico no apoio à tomada de decisão, obtendo resultados mais eficientes. Diversos cenários têm se beneficiado das utilidades do SAD, como no gerenciamento de desastres por meio de dados voluntários [Horita and de Albuquerque 2013], na área médica apresentando estimativas e previsões sobre diagnósticos ou em cidades inteligentes [Mendonça 2004], auxiliando a tomada de decisão com o auxílio de sensores e dispositivos inteligentes engenharia de tráfego entre outros desafios urbanos [Pettit et al. 2018]. Em conjunto à evolução dos SAD, a produção e disseminação de dados na rede aumentou exponencialmente [Daradkeh 2019, Al-Qirim et al. 2017]. Em decorrência do aumento no volume de dados, novos desafios surgiram, como o excesso ou a falta de dados disponíveis e decisões a serem tomadas por mais de um indivíduo [Karacapilidis 2006]. Com o objetivo de enfrentar os desafios citados, os SAD estão implementando Visual Analytics (VA) com a finalidade de descobrir novos conhecimentos em grandes volumes de dados. VA utiliza o poder computacional e as capacidades cognitivas para obtenção de insights nos grandes volumes de dados [Keim et al. 2008].

Embora a implementação do VA nos SAD esteja presente em contextos diversos [Eaglin et al. 2015, Al-Serafi and Elragal 2014, Park et al. 2016], a ausência de métodos de representação, desenvolvimento e avaliação formais na literatura pode dificultar o desenvolvimento e manutenção de SADs que utilizaram VA, tendo em vista que os sistemas são desenvolvidos, avaliados e representados informalmente [Cook et al. 2015, Wu et al. 2016, González-Torres et al. 2019, Gonzales and Horita 2020]. Desta forma, é relevante que se estudem métodos de representação de SAD e VA e que se indetifique as características de VA encontradas nas arquiteturas dos SAD.

Neste sentido, esta pesquisa visa contribuir apresentando uma taxonomia de arquiteturas de SAD baseada em VA, com a finalidade de identificar quais são as principais características do VA dentro de uma arquitetura de SAD, para que posteriormente sejam estabelecidos padrões de arquitetura e desenvolvimento.

\section{Apresentação do Problema}

SAD é uma classe de sistema que tem acompanhado o desenvolvimento das organizações desde a década de 70. Podemos tomar por definição de que um SAD auxilia na tomada de decisão baseando-se em dados empíricos, nos quais é possível adquirir conhecimento mais detalhado sobre a decisão a ser tomada e as possibilidades existentes. A evolução tecnológica trouxe novos desafios para os SAD, como o excesso ou a falta de dados disponíveis e decisões a serem tomadas por mais de um indivíduo [Karacapilidis 2006]. Com o objetivo de enfrentar os desafios citados, os SAD estão implementando Visual Analytics (VA) com a finalidade de descobrir novos conhecimentos em grandes volumes de dados. VA utiliza o poder computacional e as capacidades cognitivas para obtenção de insights nos grandes volumes de dados [Keim et al. 2008].

Embora o VA tenha despertado interesse por parte da comunidade acadêmica, evoluindo desde 2005, e trazendo bons resultados em conjunto com os SAD, a literatura sobre a utilização de VA em SAD não apresenta características ou especificações de como são implementados. Uma das formas que a ciência utiliza para classificar as características de um determinado tipo de objeto é a classificação por uma taxonomia ou ontologia. Como principais exemplos de taxonomias sobre SAD, podemos citar [Pearson and Shim 1994, Pearson and Shim 1995, Power 2004]. Tais taxonomias não 
apresentam quais formas o VA é implementado, enquanto que as taxonomias de VA [Thomas 2009, Greitzer et al. 2011, Visual Analytics Community 2010] não apresentam orientações para implementação de VA. Esta ausência na classificação acaba por não permitir a identificação de práticas uniformes de desenvolvimento e avaliação, como apontado por [Cook et al. 2015, González-Torres et al. 2019].

Tendo em vista a importância e a relevância que o VA tem ganhado nos SAD, surge a seguinte problemática: Quais características de VA são encontradas nas arquiteturas de um SAD?

\section{Proposta de Solução e Método de Pesquisa}

De acordo com o exposto na seção anterior, esta pesquisa tem a hipótese de que uma taxonomia de arquiteturas de SAD baseadas em VA é capaz de identificar e classificar características utilizadas, permitindo identificar quais estruturas são utilizadas na implementação do VA. Assim, o principal objetivo desta pesquisa é identificar as características arquiteturais de acordo com a taxonomia. Escolheu-se o domínio dos SAD, pois um mapeamento sistemático realizado previamente indicou a existência desta lacuna. Para o desenvolvimento desta pesquisa, foram estabelecidos três principais etapas:

- Mapeamento sistemático da literatura na temática de SAD, VA e Arquiteturas de Software de acordo com [Petersen et al. 2008, Kitchenham et al. 2009];

- Elaboração da Taxonomia de acordo com o método de Nickerson et al [Nickerson et al. 2013];

- Avaliação das características arquiteturais apontadas pela taxonomia com base no GQM (Goal-Question-Metric) [Basili 1994].

\section{Projeto de Avaliação da Solução}

A avaliação e validação da taxonomia é dada em duas etapas, sendo a primeira pela aplicação da taxonomia, na classificação de um conjunto de objetos, sendo parte integrante do método de Nickerson et al, enquanto que a segunda etapa de validação será por meio de um questionário aplicado à profissionais e pesquisadores da área para avaliação das características arquiteturais encontradas pela taxonomia. A elaboração do questionário terá como base o GQM.

\section{Atividades Realizadas}

Como atividades já realizadas, publicou-se um mapeamento sistemático, nas temáticas de VA, SAD e arquiteturas de software, onde foram analisados 642 trabalhos no período de 2001 até 2019, sendo que destes, 50 trabalhos foram relacionados à temática da pesquisa. O mapeamento identificou algumas lacunas de pesquisa na área, sendo que esta pesquisa se baseou na necessidade de se pesquisar meios eficazes da incorporação do VA nas arquiteturas do SAD. A taxonomia foi desenvolvida a partir dos trabalhos selecionados pelo mapeamento, utilizando as características identificadas para agrupamento das arquiteturas. Como última etapa, a taxonomia precisa ser avaliada sob a ótica de profissionais e especialistas da área, onde as características arquiteturais identificadas serão avaliadas quanto sua aplicabilidade em cenários reais. 


\section{Conclusão}

Esta pesquisa propõe uma taxonomia de arquiteturas de SAD baseadas em VA, com a finalidade de apoiar a análise arquitetural por meio da classificação e descrição das características de arquitetura.

Com base no processo de análise arquitetural, esperamos que a taxonomia possa ser incluída como uma atividade, no apoio da classificação e descrição de características arquiteturais que já foram utilizadas na literatura, criando assim um primeiro passo no desenvolvimento sistemático de sistemas de VA. Por meio desta pesquisa, poderão ser estudados meios de avaliação mais eficientes. As futuras linhas do trabalho devem se concentrar em expandir o processo de análise arquitetural para sistemas que utilizem VA de uma forma mais ampla, e com base nesta taxonomia estabelecer padrões de projeto que abordem problemas comuns com relação a implementação dos elementos do processo de VA (Visualização, Conhecimento, Modelos e Dados), identificando sistemáticas de elementos estruturais.

\section{Referências}

Al-Qirim, N., Tarhini, A., and Rouibah, K. (2017). Determinants of big data adoption and success. In Proceedings of the International Conference on Algorithms, Computing and Systems, ICACS '17, page 88-92, New York, NY, USA. Association for Computing Machinery.

Al-Serafi, A. and Elragal, A. (2014). Visual trajectory pattern mining: An exploratory study in baggage handling systems. In Advances in Data Mining. Applications and Theoretical Aspects, pages 159-173, St. Petersburg. Springer International Publishing.

Basili, V. (1994). GQM approach has evolved to include models. IEEE Software, 11(1).

Cook, K. A., Scholtz, J., and Whiting, M. A. (2015). A software developer's guide to informal evaluation of visual analytics environments using vast challenge information. In 2015 IEEE Conference on Visual Analytics Science and Technology (VAST), pages 193-194, Chicago, USA. IEEE, IEEE.

Daradkeh, M. K. (2019). Determinants of visual analytics adoption in organizations. Information Technology \& People.

Eaglin, T., Wang, X., Ribarsky, W., and Tolone, W. (2015). Ensemble visual analysis architecture with high mobility for large-scale critical infrastructure simulations. In Visualization and Data Analysis 2015, volume 9397, page 939706, San Francisco CA, USA. International Society for Optics and Photonics, SPIE.

Gonzales, G. R. and Horita, F. E. A. (2020). Visual analytics in decision support system architecture: A systematic mapping study. In Proceedings of the XIX Brazilian Symposium on Human Factors in Computational Systems, pages 140-149, New York, NY, USA. ACM.

González-Torres, A., Navas-Sú, J., Hernández-Vásquez, M., Hernández-Castro, F., and Solano-Cordero, J. (2019). A visual analytics architecture for the analysis and understanding of software systems. Enfoque UTE, 10(1):218-233. 
Greitzer, F. L., Noonan, C. F., and Franklin, L. (2011). Cognitive foundations for visual analytics. Technical report, Pacific Northwest National Lab.(PNNL), Richland, WA (United States).

Horita, F. E. and de Albuquerque, J. P. (2013). An approach to support decision-making in disaster management based on volunteer geographic information (vgi) and spatial decision support systems (sdss). In ISCRAM.

Karacapilidis, N. (2006). An overview of future challenges of decision support technologies. Intelligent Decision-making Support Systems, pages 385-399.

Keim, D. A., Mansmann, F., Schneidewind, J., Thomas, J., and Ziegler, H. (2008). Visual analytics: Scope and challenges. In Visual data mining, pages 76-90. Springer, Berlin, Germany.

Kitchenham, B., Brereton, O. P., Budgen, D., Turner, M., Bailey, J., and Linkman, S. (2009). Systematic literature reviews in software engineering-a systematic literature review. Information and software technology, 51(1):7-15.

Mendonça, E. A. (2004). Clinical decision support systems: perspectives in dentistry. Journal of dental education, 68(6):589-597.

Nickerson, R. C., Varshney, U., and Muntermann, J. (2013). A method for taxonomy development and its application in information systems. European Journal of Information Systems, 22(3):336-359.

Park, H., Bellamy, M. A., and Basole, R. C. (2016). Visual analytics for supply network management: System design and evaluation. Decision Support Systems, 91:89-102.

Pearson, J. M. and Shim, J. (1994). An empirical investigation into decision support systems capabilities: a proposed taxonomy. Information \& Management, 27(1):4557.

Pearson, J. M. and Shim, J. (1995). An empirical investigation into dss structures and environments. Decision Support Systems, 13(2):141-158.

Petersen, K., Feldt, R., Mujtaba, S., and Mattsson, M. (2008). Systematic mapping studies in software engineering. In Ease, volume 8, pages 68-77, Swindon, United Kingdom.

Pettit, C., Bakelmun, A., Lieske, S. N., Glackin, S., Thomson, G., Shearer, H., Dia, H., Newman, P., et al. (2018). Planning support systems for smart cities. City, culture and society, 12:13-24.

Power, D. J. (2004). Specifying an expanded framework for classifying and describing decision support systems. Communications of the Association for Information Systems, 13(1):13.

Thomas, J. (2009). Taxonomy for visual analytics: Seeking feedback. VAC Views, May.

Visual Analytics Community (2010). Visual Analytics Taxonomy Draft Taxonomy for Visual Analytics. http://vacommunity.org/Visual+Analytics+ Taxonomy. Online; accessed 5 May 2020.

Wu, Y., Cao, N., Gotz, D., Tan, Y.-P., and Keim, D. A. (2016). A survey on visual analytics of social media data. IEEE Transactions on Multimedia, 18(11):2135-2148. 